\title{
Characterization of the bacterial microbiota composition and evolution at different intestinal tract in wild pigs (Sus scrofa ussuricus)
}

\author{
Guang-li Yang ${ }^{\text {Corresp., Equal first author, } 1}$, Chuan-xin Shi Equal first author, 1 , Shu-hong Zhang ${ }^{1}$, Yan Liu ${ }^{2}$, Zhi-qiang Li ${ }^{1}$, Feng-yi Gao \\ , Yan-yan Cui ${ }^{1}$, Yong-feng Yan ${ }^{1}$, Ming Li ${ }^{\text {Corresp. } 3}$ \\ 1 Department of Biology and Food Sciences, Shangqiu Normal University, Shangqiu city, Henan Province, China \\ 2 College of Animal Husbandry Engineering, Henan Vocational College of Agricultural, Zhengzhou city, Henan Province, China \\ 3 Engineering College of Animal Husbandry and Veterinary Science, Henan Agricultural University, Zhengzhou city, Henan Province, China \\ Corresponding Authors: Guang-li Yang, Ming Li \\ Email address: guangliyang@163.com, liming@henau.edu.cn
}

Commensal microorganisms are essential to the normal development and function of many aspects of animal biology, including digestion, nutrient absorption, immunological development, behaviors, and evolution. The specific microbial composition and evolution of the intestinal tracts of wild pigs remain poorly characterized. This study therefore sought to assess the composition, distribution, and evolution of the intestinal microbiome of wild pigs. For these analyses, 16S rRNA V3-V4 regions from 5 gut sections prepared from each of 3 wild sows were sequenced to detect the microbiome composition. These analyses revealed the presence of 6,513 operational taxonomic units (OTUs) mostly distributed across 17 phyla and 163 genera in these samples, with Firmicutes and Actinobacteria being the most prevalent phyla of microbes present in cecum and jejunum samples, respectively. Moreover, the abundance of Actinobacteria in wild pigs was higher than that in domestic pigs. At the genus level the Bifidobacterium and Allobaculum species of microbes were most abundant in all tested gut sections, with higher relative abundance in wild pigs relative to domestic pigs, indicating that in the process of pig evolution, the intestinal microbes also evolved, and changes in the intestinal microbial diversity could have been one of the evolutionary forces of pigs. Intestinal microbial functional analyses also revealed the microbes present in the small intestine (duodenum, jejunum, and ileum) and large intestine (cecum and colon) of wild pigs to engage distinct metabolic spatial structures and pathways relative to one another. Overall, these results offer unique insights that would help to advance the current understanding of how the intestinal microbes interact with the host and affect the evolution of pigs. 


\section{Characterization of the bacterial microbiota composition and}

\section{2 evolution at different intestinal tract in wild pigs (Sus scrofa}

\section{3 ussuricus)}

4 Guangli Yang ${ }^{1, *}$, Chuanxin Shi ${ }^{1, *}$, Shuhong Zhang ${ }^{1}$, Yan $\mathrm{Liu}^{2}$, Zhiqiang $\mathrm{Li}^{1}$, Fengyi Gao ${ }^{1}$, Yanyan Cui ${ }^{1}$,

5 Yongfeng $\mathrm{Yan}^{1}$, Ming $\mathrm{Li}^{3}$

$6{ }^{1}$ Department of Biology and Food Sciences, Shangqiu Normal University, 476000 Shangqiu, China

$7 \quad{ }^{2}$ College of Animal Husbandry Engineering, Henan Vocational College of Agricultural, 450002 Zhengzhou,

8 China

9 'Engineering College of Animal Husbandry and Veterinary Science, Henan Agricultural University,

10450002 Zhengzhou, China

12 Corresponding Author:

13 Ming Li

14 Engineering College of Animal Husbandry and Veterinary Science, Henan Agricultural University, 450002

15 Zhengzhou, China

16 Email address: liming@henau.edu.cn

17 Guangli Yang ${ }^{1}$

18 Department of Biology and Food Sciences, Shangqiu Normal University, 476000 Shangqiu, China

19 Email address: guangliyang@163.com

21 *These authors are as co-first authors 
23 Abstract: Commensal microorganisms are essential to the normal development and function of

many aspects of animal biology, including digestion, nutrient absorption, immunological development, behaviors, and evolution. The specific microbial composition and evolution of the intestinal tracts of wild pigs remain poorly characterized. This study therefore sought to assess the composition, distribution, and evolution of the intestinal microbiome of wild pigs. For these analyses, 16S rRNA V3-V4 regions from 5 gut sections prepared from each of 3 wild sows were sequenced to detect the microbiome composition. These analyses revealed the presence of 6,513 operational taxonomic units (OTUs) mostly distributed across 17 phyla and 163 genera in these samples, with Firmicutes and Actinobacteria being the most prevalent phyla of microbes present in cecum and jejunum samples, respectively. Moreover, the abundance of Actinobacteria in wild pigs was higher than that in domestic pigs. At the genus level the Bifidobacterium and Allobaculum species of microbes were most abundant in all tested gut sections, with higher relative abundance in wild pigs relative to domestic pigs, indicating that in the process of pig evolution, the intestinal microbes also evolved, and changes in the intestinal microbial diversity could have been one of the evolutionary forces of pigs. Intestinal microbial functional analyses also revealed the microbes present in the small intestine (duodenum, jejunum, and ileum) and large intestine (cecum and colon) of wild pigs to engage distinct metabolic spatial structures and pathways relative to one another. Overall, these results offer unique insights that would help to advance the current understanding of how the intestinal microbes interact with the host and affect the evolution of pigs.

Keywords: Wild pigs; Gut microbiota; Structure and composition; 16S rRNA gene; Evolution

\section{Introduction}

The intestines of mammals are colonized by trillions of microorganisms wherein they play a dynamic role in controlling host physiological, immunological, and digestive processes (Brestoff \& Artis, 2013). The vertebrate intestinal microbiome plays a key role in regulating host biology, and further research suggests it may also influence vertebrate evolution (Sharpton, 2018). Recent years have witnessed a growing number of studies focusing on the composition of the porcine intestinal microbiota (Crespo-Piazuelo et al., 2018; Gao et al., 2019; Kelly et al., 2017; Xiao et al., 2018; Yang et al., 2016; Zhao et al., 2015), with correlations having been drawn between this composition and pig average daily weight gain (Mach et al., 2015; Ramayo-Caldas et al., 2016), feed conversion efficiency and intake (Camarinha-Silva et al., 2017; McCormack et al., 2017; Quan et al., 2018, 2019). A few studies have focused on the microbial makeup in distinct regions of the intestinal tract. Furthermore, these studies have largely been restricted to certain breeds of domestic pigs such as the Large White (Zhao et al., 2015), Laiwu (Yang et al., 2016), Gloucestershire Old Spot (Kelly et al., 2017), Lberian pigs (Crespo-Piazuelo et al., 2018), Jinhua and Landrace (Xiao et al., 2018), and Chinese Shanxi Black breeds (Gao et al., 2019). 
61 Wild pigs are the most closely-related species to domestic pigs, with the divergence

62 between these two populations having been initiated in Eurasia roughly 10,000 years ago (Larson

63 et al., 2005). Domestic pigs are now distinct from their wild counterparts in various ways, such

64 as phenotype, as evidenced by coat color differences (Yang et al., 2019). The specific microbial

65 composition and evolution of the intestinal tracts of wild pigs, however, remain poorly

66 characterized. Many studies have found that at different evolutionary stages, vertebrate species

67 exhibit distinct patterns of microbiota diversity and functional that are correlated with the evolutionary history of that species (Brooks et al., 2016; Gaulke et al., 2017; Groussin et al., 2017; Ley et al., 2008). There is also evidence to support the fact that microbial signatures may be heritable (Koskella et al., 2017), and that there are microbial and host co-phylogenetic patterns owing to their close reliance on one another (Brooks et al., 2016; Gaulke et al., 2017; Groussin et al., 2017; Moeller et al., 2016). These results are consistent with a model wherein vertebrate evolution is influenced by the composition of the gut microbiome (Gaulke et al., 2017; Groussin et al., 2017; Ley et al., 2008; Muegge et al., 2011), with many processes having the potential to drive or shape this relationship (Brooks et al., 2016). To date, uncertainty prevails as to how significantly the gut microbiome diversity affects vertebrate evolution.

Recent, next generation sequencing has provided more 16S rRNA gene sequence reads that can be further analyzed to provide in depth studies microbial populations and compositions, and to facilitate more complex analyses of the link between the microbiome and vertebrate evolution. Efforts to assess the functional capabilities of the microbiota in a given organism have been supported by metagenomics annotation efforts (Nayfach et al., 2015). The studies in rodents have demonstrated that microbiome transplantation can alter feed efficiency in these animals (Brooks et al., 2016), suggesting that certain microbial phyla are optimized to support host growth and survival, thus tying the microbiome directly to key evolutionary processes. When inbred mice maintained in laboratory facilities receive feces transplant from wild mice, improving their resistance, it suggests that the artificial inbreeding might have led to microbiome drift toward a less resilient phylotype (Rosshart et al., 2017). Additional research has identified bacteria capable of degrading oxalate in the gut of rodents, suggesting that these bacteria support the adaptation of these animals to additional dietary niches by expanding their ability to utilize certain nutrient sources (Kohl et al., 2014). The intestinal microbiota of domestic pigs, wild pigs, and Red river hogs studies reported that the Lactobacilli bacteria are primarily present in domesticated or captured pigs, whereas Bifidobacterium dominates the intestinal microbiota of wild animals (Ushida et al., 2016).

Further studies of the gut microbiome of wild pigs may, therefore, offer valuable insight into the relationship between this microbial community and porcine evolution. As such, in the present study we sampled the gut microbiome in five distinct regions of the gastrointestinal tract of wild pigs [duodenum (DU), jejunum (JE), ileum (IL), cecum (CE), and colon(CO)], using 15 samples for $16 \mathrm{~S}$ rRNA sequencing to facilitate microbial characterization. Using comparative 
99 analyses stemming from this approach, we sought to advance the current understanding of the

100 porcine microbiome and its association with the evolution of these animals.

\section{Materials and Methods}

\section{Animal sample collection.}

103 We selected three unrelated (no shared ancestors for 3+ generations) female adult (4-year-old)

104 wild pigs from populations in Xingyang county of Henan province in China that were derived

105 from similar genetic backgrounds and subjected to comparable husbandry practices. These

106 animals were fed twice daily with a controlled diet composed of corn and soybean and

107 supplemented with hay, which likely facilitated reductions in microbiota variability relative to

108 wild animal populations. Animals had free access to water, and all were healthy and not

109 subjected to any antibiotic treatments. After sacrificing these three animals, the gastrointestinal

110 tract was removed from each animal within 30 minutes of death and luminal contents were

111 collected from each of the 5 indicated segments. These contents were specifically collected from

112 the middle of each sample, with full disinfection of the experimental tools and work area being

113 performed between samples to prevent any microbial cross-contamination. Samples were then

114 frozen in liquid nitrogen and stored at $-80^{\circ} \mathrm{C}$. The animal care and use guidelines put forth by

115 the Ministry of Science and Technology of China (Guidelines on Ethical Treatment of

116 Experimental Animals (2006) No. 398) were followed for this study, with the Ethics Committee

117 of Shangqiu Normal University having approved all experiments herein (Shang (2017) No. 168).

118 16S rRNA gene sequencing.

119 A TIANamp Stool DNA Kit (DP328; Tiangen BioTech Beijing, China) was used to isolate

120 luminal bacterial DNA based on provided directions, after which a NanoDrop One Microvolume

121 Spectrophotometer (Thermo Fisher Scientific, DE, USA) was used to assess DNA

122 concentrations, while $0.8 \%$ agarose gel electrophoresis was used to assess DNA quality and

123 purity. The bacterial 16S rRNA V3-V4 region was amplified using the well-documented primer

124 pair: 338F (5'- ACTCCTACGGGAGGCAGCA -3') and 806R (5'-

125 GGACTACHVGGGTWTCTAAT -3'). To facilitate multiplexed sequencing, samples were

126 barcoded with 7-bp tags that were specific for each sample. Individual PCR reactions were

127 composed of $5 \mu \mathrm{L}$ Q5 reaction buffer $(5 \times), 5 \mu \mathrm{L}$ Q5 High- Fidelity GC buffer $(5 \times), 0.25 \mu \mathrm{L}$ Q5

128 High-Fidelity DNA Polymerase $(5 \mathrm{U} / \mu \mathrm{L}), 2 \mu \mathrm{L}(2.5 \mathrm{mM}) \mathrm{dNTPs}, 1 \mu \mathrm{L}(10 \mathrm{uM})$ of each primer, 2

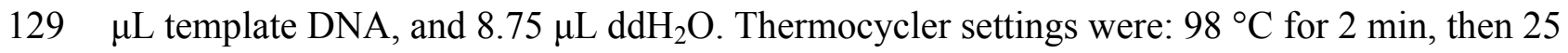

130 cycles of $98^{\circ} \mathrm{C}$ for $15 \mathrm{~s}, 55^{\circ} \mathrm{C}$ for $30 \mathrm{~s}$, and $72{ }^{\circ} \mathrm{C}$ for $30 \mathrm{~s}$, followed by a final of $5 \mathrm{~min}$ at $72{ }^{\circ} \mathrm{C}$.

131 Phusion High-Fidelity PCR Master Mix (New England BioLabs, MA, USA) was used for PCR

132 reactions. Next, $2 \%$ agarose gel electrophoresis was used to confirm that amplicon sizes were

133 consistent with expectations ( $\sim 50 \mathrm{bp}$ ) and that samples were of good quality and purity. Then,

134 the $\sim 500$ bp sample band was subjected purification with a GeneJET Gel Extraction Kit (Thermo

135 Scientific, USA) per the manufacturer's instructions. Purified amplicons were used for library

136 preparation. We used a Next Ultra DNA Library Prep Kit for Illumina (New England BioLabs)

137 for library preparation per the manufacturer's instructions, with a Qubit@2.0 Fluorometer 
138 (Thermo Fischer Scientific) and an Agilent Bioanalyzer 2100 machine used to evaluate library

139 quality. Sequencing of the resultant library was performed on an Illumina MiSeq platform,

140 generating 250-bp paired-end reads.

141

\section{Sequence Analysis}

143 Sequencing data was processed using the Quantitative Insights Into Microbial Ecology (QIIME, 144 v1.8.0) pipeline (Caporaso et al., 2010). Briefly, any sequencing reads that exactly matched 145 barcodes were assigned as valid sequences to the corresponding samples. Any low-quality reads 146 were then filtered to remove reads meeting the following criteria: sequences that were $<150 \mathrm{bp}$ 147 long, had average Phred scores $<20$, contained ambiguous bases, or mononucleotide repeats $>8$ 148 bp long. FLASH was used for paired-end read assembly (Magoc et al., 2011). High-quality 149 sequences that remained after chimera detection were grouped using UCLUST into operational 150 taxonomic units (OTUs) at the $97 \%$ sequence identity level (Edgar et al., 2011). For each OTU, a 151 representative sequence was then selected on the basis of default parameters to facilitate

152 BLAST-mediated taxonomic classification with the Greengenes Database (13.8 version;

153 DeSantis et al., 2006). The abundance of the OTUs in a given sample were compiled in an OTU 154 table. Any OTUs containing $<0.001 \%$ of total sequences across all samples were discarded. To 155 minimize the impact of variable sequencing depth among samples, we generated a rounded 156 rarefied OTU table by taking the average of 100 evenly resampled OTU subsets under the $90 \%$ 157 of the minimum sequencing depth for further analyses.

\section{Bioinformatics and statistical analysis.}

159 QIIME and R packages (v3.2.0) were used to analyze sequencing data. We used the Chao1, 160 Abundance-based coverage estimator (ACE) metric, Shannon, and Simpson alpha diversity 161 indices, which were calculated using QIIME (Caporaso et al., 2010), to assess species diversity 162 and complexity among samples. The richness and evenness of OTU distributions across samples were assessed based upon OTU-level ranked abundance curves. UniFrac distance metrics were used to assess variations in beta diversity among samples corresponding to structural differences in microbial community composition (Lozupone \& Knight, 2005; Lozupone, Hamady, Kelley \& Knight, 2007), and these metrics were visualized using nonmetric multidimensional scaling (NMDS) and unweighted pair-group method with arithmetic means (UPGMA) hierarchical clustering (Ramette, 2007). The abundance of microbes at the phylum and genus levels were compared between samples with Metastats (White et al., 2009).

In order to identify those OTUs differing significantly among the five sample regions, we utilized the linear discriminant analysis coupled with the effect size (LEfSe) algorithm based upon relative OTU abundance (Segata et al., 2011). Briefly, this algorithm first used a nonparametric factorial Kruskal-Wallis (KW) sum-rank test to identify OTUs that were present at significantly different levels, after which pairwise Wilcoxon tests were used to assess biological consistency between groups. LDA scores were then used to yield an estimated effect size for 
176 each differentially abundant feature. PICRUSt was used to predict microbial function according

177 to high-quality sequences (Langille et al., 2013).

\section{Results}

179 Sequencing data overview

180 We were able to successfully amplify the 16S rRNA sequences from luminal samples collected

181 from 5 different gut sections from each of three 4-year-old wild pigs. All 15 of the resultant

182 samples were sequenced, yielding 925,293 reads that clustered into 687,477 tags (Supplementary

183 Table S1). The sequenced raw data sets have been submitted in the NCBI databases

184 (PRJNA575288). QIIME processing grouped these samples into 6,513 operational taxonomic

185 units (OTUs; Supplementary Table S2), and we then performed species accumulation and rank-

186 abundance curve analyses to confirm the presence of these OTUs within each of our samples.

187 Species accumulation and rank-abundance curve patterns were similar across samples,

188 suggesting that most detectable bacterial species were present in most or all samples (Fig. 1A

189 and B).

190 Microbiota composition throughout the intestinal tract of wild pigs.

191 To explore the composition of microbial communities in different regions of the intestinal tract

192 of wild pigs, we initially assessed the alpha diversity of the microbiome in the 5 tested regions

193 (Supplementary Table S3). We noted significant differences in the average number of OTUs

194 among these sections, with the highest number of OTUs being present in the duodenum (1904 \pm

195 38) relative to the jejunum (1381 \pm 97$)$ and ileum (1377 $\pm 46 ; P<0.05)$, and with higher OTU

196 numbers in cecum samples $(1955 \pm 138)$ relative to jejunum samples $(1381 \pm 97 ; P<0.05)$. We

197 then calculated the ACE and Chaol indices, which respectively measure richness/evenness and

198 richness. Samples from the ileum and jejunum had significantly lower ACE and Chao1 values

199 than did samples from the duodenum, cecal, and colon ( $P<0.05$; Fig. $2 \mathrm{~A}$ and B). We further

200 used the Shannon index as an additional comparison of alpha diversity analyzing numbers of

201 species and relative abundance within a given sample, but no significant differences in this index

202 were detected among regions of the intestinal tract $(P>0.05$; Fig. $2 \mathrm{C})$. Similarly, no differences

203 in the Simpson index were detected among samples $(P>0.05$; Fig. 2D). We further explored

204 how similar or variable the microbial community composition was between samples at the OTU

205 level via NMDS and UPGMA approaches. As shown in Figures 3A and 3B (Unweighted

206 UniFrac), we observed significant differences in gut microbiota composition across sample

207 regions, with the bacterial composition in samples from the duodenum, jejunum, and ileum

208 differing significantly from cecal and colon samples, which were similar to one another (Fig.

209 3A). A Bray Curtis clustering analysis revealed that the majority of gut microbes clustered into

210 two subgroups, with the cecum and colon samples clustering separately from the ileum,

211 duodenum, and jejunum samples via UPGMA (Fig. 3B). Together these findings clearly

212 indicated that the composition of the gut microbiome was not uniform throughout the intestinal

213 tract of wild pigs, with cecal and colon samples being more similar to one another than were

214 duodenal, jejunal, and ileal samples. 
215

216

217

218

219

220

221

222

223

224

225

226

227

228

229

230

231

232

233

234

235

236

237

238

239

240

241

242

243

244

245

246

247

248

249

250

251

252

253

We additionally assessed the taxonomic distributions of the most abundant bacterial OTUs in each sample region. Based on the bacterial relative abundance of the top 17 phyla, we specified about the Firmicutes levels at each location or made it clear in which location that Firmicutes were the most prevalent phylum (52.35\% of cecal microbes), followed by Actinobacteria (46.66\% of jejunal microbes). In contrast, Proteobacteria levels in the cecal and colon samples were low (1.10\% and $0.92 \%$, respectively). The relative Bacteroidetes abundance in duodenal, jejunal, ileal, cecal, and colon samples was $11.60 \%, 0.40 \%, 5.10 \%, 9.73 \%$, and $16.86 \%$, respectively. We also detected Cyanobacteria $(3.10 \%)$ in the duodenum, and Verrucomicrobia (1.35\%) in the colon (Fig. 4A; Supplementary Table S4).

At the genus level, a total of 163 genera were identified. Bifidobacterium was most prevalent and the relative abundance was $34.87 \%, 31.10 \%, 17.21 \%, 22.48 \%$, and $22.70 \%$ in the duodenum, jejunum, ileum, cecum, and colon, respectively. In addition, we also observed Lactobacillus (11.63\%), Prevotella (9.97\%), Unclassified_Clostridiaceae (7.62\%), Unclassified_Coriobacteriaceae (6.51\%), and Megasphaera (4.04\%) in the duodenum. Unclassified_Coriobacteriaceae (13.52\%), Psychrobacter (12.39\%), Lactobacillus (11.05\%), and Allobaculum (4.73\%) were most prevalent in the jejunum. Psychrobacter $(17.50 \%)$, Unclassified_Coriobacteriaceae (10.93\%), Allobaculum (4.26\%), Unclassified_Clostridiaceae (5.58\%), Prevotella (4.29\%), and Unclassified_Moraxellaceae (5.98\%) were most prevalent in the ileum. Allobaculum (14.970\%), Unclassified_Coriobacteriaceae (12.12\%),

Unclassified_Clostridiaceae (11.90\%), Unclassified_Clostridiales (5.91\%), Unclassified_Lachnospiraceae (4.96\%), Unclassified_Ruminococcaceae (6.41\%), and Unclassified_Bacteroidales (6.28\%) were the most prevalent in the cecum, Unclassified_Coriobacteriaceae (6.80\%), Allobaculum (12.80\%), Unclassified_Clostridiaceae (5.21\%), Unclassified_Clostridiales (4.84\%), Unclassified_Lachnospiraceae (4.80\%), Unclassified_Ruminococcaceae (5.21\%), and Wautersiella (11.15\%) were the most prevalent in the colon (Fig. 4B; Supplementary Table S5). Furthermore, we found in the distribution of 33 genera of wild pig intestinal microbiota that the abundance of only two genera in the ileum is 0 , the average distribution index of each genera in the ileum is the lowest, and the ileum microbiome presents a greater evenness than that at other locations. These results demonstrated the presence of many genera of bacteria across the different regions of the gut, with more uniform distribution in the ileum.

To identify the bacterial species most characteristic of the five tested gut regions, we conducted an LEfSe analysis of the taxa with LDA scores $>2$; this approach revealed that 28 OTUs were differentially present in the ileal, cecal, and colon samples (Fig. 5, Supplementary Table S6). The relative abundance of 14 OTUs was evident in the colon samples relative to those from the ileum and cecum; these OTUs included the Verrucomicrobia, s24_27, rfp12, WCHB1_41,Verruco_5, Ruminococcus, p_75_a5, CF231, Christensenellaceae, Verrucomicrobiae, Akkermansia, Dorea, Verrucomicrobiales, and Verrucomicrobiaceae genera. We noted a relatively higher Actinomycetales abundance in the ileum relative to the other gut 
254 locations. In the cecum, 13 OTUs were present with higher abundance than that in other gut

255 regions, with one representative OTU among these 13 being Ruminococcaceae (Fig. 5).

256 Functional analysis of the gut microbiota along the intestine tract.

257 We next used PICRUSt to develop an understanding of the metagenomic activity of the

258 identified bacteria across our samples, as such functional assessments of the microbiome may

259 offer more meaningful insights into the spatial distinctions in metabolic activity across the length

260 of the intestinal tract. These metagenomic inferences were made based upon available

261 annotations for the detected OTUs in this study. The genes identified through this metagenomic

262 analysis were then aligned to the KEGG database to gain functional insights. Through this

263 approach, we identified 5945 KEGG genes (Supplementary Table S7) that were assigned to 289

264 pathways (Supplementary Table S8). We then assessed the relative abundance of these pathways

265 among samples from different gut regions $(P<0.05$; Fig. 6), identifying 15 significantly

266 differentially enriched pathways (Fig. 7). A total of 10 pathways were significantly enriched in

267 ileal samples (Caprolactam_degradation, Glutathione_metabolism, Benzoate_degradation,

268 Cytochrome_P450, Drug_metabolism_cytochrome_P450,

269 Metabolism_of_xenobiotics_by_cytochrome_P450, Alzheimer_disease, Parkinson_disease,

270 Cardiac_muscle_contraction, Apoptosis), while 3 were more enriched in cecal samples

271 (Methane_metabolism, Epithelial_cell_signaling_in_Helicobacter_pylori_infection,

272 Germination), and 2 were more enriched in jejunal samples (Tyrosine_metabolism,

273 Chloroalkane_and_chloroalkene_degradation. The pathways that were enriched in ileum samples

274 were associated with glycerophospholipid metabolism (ko02029), diterpenoid biosynthesis

275 (ko09686), and bacterial chemotaxis (ko02030). The pathways enriched in cecum and colon

276 samples were linked with steroid hormone biosynthesis (ko03088), the pentose phosphate

277 pathway (ko00615), and arginine and proline metabolism (ko01990, ko01992). The pathways

278 enriched in jejunum samples were associated with fatty acid metabolism (ko01897 and

279 ko04924), ABC transporters (ko02006, ko02008), the biosynthesis of unsaturated fatty acids

280 (ko02050), and cardiovascular diseases (ko05410; Fig. 8; Supplementary Table S9). Together

281 these findings suggest that the functional metabolic activity of the microbiome varies over the

282 length of the intestinal tract, with certain pathways being preferentially engaged in a spatially-

283 defined manner.

284 Discussion

285 High throughput sequencing analyses have facilitated rapid advances in our understanding of the

286 intestinal microbiome over the last decade, facilitating both functional and compositional

287 analyses of this complex microbial community. The specific microbial composition and

288 evolution of the intestinal tracts of wild pigs, however, remain poorly characterized. As such, we

289 utilized 16S rRNA sequencing to survey the composition, distribution, and function of intestinal

290 microbiome across different regions of the digestive tract in Chinese wild pigs. In addition,

291 previous studies have not investigated the link between the gut microbiome and porcine

292 evolution. Hence, in this study, we also inferred the relationship between variations in the 
293 porcine gut microbiota compositional and pig evolution, providing novel insights into the

294 evolution of pigs.

295 The primary findings of this study centered on exploring the structural diversity of the

296

297

298

299

300

301

302

303

304

305

306

307

308

309

310

311

312

313

314

315

316

317

318

319

320

321

322

323

324

325

326

327

328

329

330

331

intestinal microbiome in wild pigs. We found that the bacterial composition of all tested samples was dominated by Firmicutes (30.45\%-52.35\%), Actinobacteria (31.22\%-46.66\%), Proteobacteria (0.92\%-32.39\%), and Bacteroidetes ( $0.40 \%-16.86 \%)$ at the phylum level (Fig. 4A and Supplementary Table S4). This was in contrast to previous studies of the intestinal microbiome of domestic pigs (Crespo-Piazuelo et al., 2018; Gao et al., 2019; He et al., 2016; Ivarsson et al., 2014; Kraler et al., 2016; Liu et al., 2012; Mach et al., 2015; Quan et al., 2018, 2019; Ramayo-Caldas et al., 2016; Slifierz et al., 2015; Xiao et al., 2018; Yang et al., 2016), which found the core microbiome in these animals to be dominated by Firmicutes and Bacteroidetes. In contrast, we found Actinobacteria to be the second most dominant bacterial phyla in the intestines of wild pigs. Thus, in this study, besides the fact that Firmicutes dominated both wild pigs and domestic pigs, Actinobacteria in wild pigs dominated and Bacteroidetes in domesticated pigs predominated. Bacteroidetes are naturally competent Gramnegative bacteria (Mell \& Redfield, 2014) and can also degrade bacterial exopolysaccharides in animal intestines (Lammerts van Bueren et al., 2015). An increasingly robust body of evidence has shown that Actinobacteria species are abundant in the intestines of animals, wherein they can produce key antibiotics, immunomodulatory compounds, and metabolites that are vital to host health and homeostasis (Matsui et al., 2012). Of note, domestic pigs are domesticated by wild pigs. During the evolution of pigs, the intestinal microbes in pigs also coevolved. From wild pig's Actinobacteria to the domestic pig Bacteroidetes phyla predominance, not only related to the pig evolution but also related to pig genetics, natural selection, environment, and feeding system. Overall, the well-documented role of intestinal Actinobacteria species as promoters of antibacterial resistance indicates that the higher levels of these bacteria in the intestines of wild pigs could correspond to improved disease resistance and roughage nutrient resistance relative to domestic pigs.

We found that Bifidobacterium and Allobaculum were the most abundant genera of bacteria in most of the tested wild pig intestinal samples, corresponding to $2.08-34.87 \%$ of total bacteria in these samples (Fig. 4B; Supplementary Table S5). However, the relative abundance of particular bacterial taxa varied substantially between different sites in the intestines. The most noticeable locations were found in the duodenum and jejunum, where Bifidobacterium relative abundance was significantly higher than in the ileum, cecum, and colon $(31.10 \%-34.87 \%$ vs 17.21\%-27.40\%; Fig. 4B; Supplementary Table S5). Allobaculum comprised over $10 \%$ of the cecal and colonic bacteria in these wild pigs, being similarly dominant to Clostridia, Psychrobacter, and Lactobacillius genera in this region of the intestine. Past studies have yielded significant variations in the genera-level intestinal microbiome composition of pigs. For example, Yang et al. (2016) found Prevotella, Lactobacillus, and Treponema to be the most abundant genera in Duroc pigs, while Xiao et al. (2017) found Prevotella, Streptococcus, and

Peer) reviewing PDF | (2019:09:41671:2:0:NEW 31 Mar 2020) 
332 SMB53 to be the most abundant genera in Hampshire pigs, with Clostridium, SMB53, and

333 Streptococcus being the most abundant in Landrace and Yorkshire pigs. This suggests that there

334 are significant differences in microbiome composition at the genus level among pigs, possibly

335 owing to differences in age, breed, feed composition, or husbandry practices.

336 In addition, Bifidobacterium species are a major probiotic species in humans, playing roles

337 in digestion, nutrient absorption and metabolism, and disease resistance owing to their ability to

338 maintain the integrity of the mucosal barrier in the intestines (Furusawa et al., 2013).

339 Bifidobacterium have, for example, been shown to help prevent rotavirus enteritis (Rigo-Adrover

340 et al., 2017) and necrotizing enterocolitis in premature rats (Satoh et al., 2016; Wu et al., 2013),

341 and to bolster immune function and inflammation in weaning rats with colitis (Izumi et al.,

342 2015). Additional research has further found that Bifidobacterium can help prevent cardiac

343 damage (Sadeghzadeh et al., 2017) and can influence the development of metabolic syndrome

344 (Bordoni et al., 2013; Kim et al., 2017; Plaza-Díaz et al., 2017(a, b); Plaza-Diaz et al., 2014; Zhu

345 et al., 2018). Short-chain fatty acid (SCFA) producing bacterial genera, including

346 Bifidobacterium and Allobaculum, have been found to provide beneficial effects to hosts through

347 these SCFAs, reducing inflammation and promoting colonic health. Similarly, Allobaculum has

348 been found to be inversely correlated with adiposity, and the abundance of these microbes

349 correspondingly increased in C57BL/6 mice fed a low-fat diet relative to those mice fed a high-

350 fat diet (Baldwin et al., 2016). We, therefore, hypothesize that the high levels of Bifidobacterium

351 and Allobaculum in the intestines of the microbiome may help to promote nutrient absorption

352 and disease resistance in wild pigs. Moreover, Ushida et al. (2016) examined the impact of

353 domestication and modern feeding practices on the intestinal microbiome composition in Suidae

354 pigs through metagenomic analyses, revealing higher relative Bifidobacterium abundance in the

355 gut of wild pigs relative to domestic pigs. They further suggested that domestication and/or

356 modern feeding practices may have led to the relative dominance of Lactobacillus that they

357 observed in the guts of domesticated pigs, as these species were only in the top 20 genera of

358 bacteria present in the guts of wild pigs. The exact factors driving this change remain uncertain,

359 and any exploration thereof necessitates the genomic analyses of Suidae-associated

360 Bifidobacterium species in an effort to identify genetic changes in these bacteria that may have

361 allowed them to adapt to growth in domesticated pigs subjected to modern feeding practices. To

362 that end, Tsuchida et al. (2017) analyzed 7 strains of Sus-associated Bifidobacterium via genomic

363 alignment ( 3 from domestic pigs and 4 from free-range wild pigs); they found that the bacterial

364 isolates from wild pigs expressed enzymes associated with fiber degradation, and the bacterial

365 isolated from domesticated animals expressed functional tetracycline-resistant genes. The

366 expression of functional tetracycline resistance genes from intestinal bacterial of domestic

367 animals could be related to the routine use of antibiotics to treat these animals during growth and

368 development. Overall, these authors observed clear differences in the gut microbiome of wild

369 pigs relative to their domestic counterparts (Ushida et al., 2016; Tsuchida et al., 2017). Together

370 these previous results suggest that over the course of porcine evolution and domestication, 
371 significant environmental and nutrient source changes are, together with artificial selection,

372 likely to result in a divergence in intestinal microbiome composition in these animals such that

373 modern domestic pigs bear a microbial cohort associated with rapid growth but poor disease

374 resistance relative to wild pigs.

375 In the final section of our study, we conducted a metagenomic analysis of the functional

376 capacity of the intestinal microbiome in wild pigs, leading us to identify 15 significantly

377 differentially enriched pathways across spatial regions within the intestine $(P<0.05$; Fig. 6). In

378 total 2 pathways were more enriched in jejunal samples, 10 were more enriched in ileal samples,

379 and 3 were more enriched in cecal samples (Fig. 7). The main metagenomic activities of the

380 jejunal microbiome in these wild pigs were related to carbohydrate metabolism via glycolysis

381 and/or gluconeogenesis. In contrast, metagenomic functions in the ileum were more closely

382 associated with fatty acid and pyruvate metabolism and xylene degradation, likely owing to the

383 abundance of Clostridiales in this region (Niu et al., 2015). The predominant metagenomic

384 functions evident in the cecum of these wild pigs were related to carbohydrate and lipid

385 metabolism, whereas protein metabolism was enriched in colonic samples. We further found a

386 preferential abundance of methane metabolism in the cecum, which is the predominant site of

387 methane generation, the relative abundance of which increases nearer to the ends of the

388 intestines. Production of SCFAs following dietary polysaccharides fermentation have been

389 previously shown to improve intestinal absorptive capacity and feed efficiency in pigs (Yang et

390 al. 2017; Pryde et al. 2002). We also found different pathways enriched in ileal, cecum, colon,

391 jejunal samples (Fig. 8; Supplementary Table S9). These metagenomic analysis results thus

392 suggest that the gut microbiome exhibits distinct functional and spatial organization that helps to

393 facilitate the rapid degradation and utilization of diverse nutrient sources by local bacterial

394 species that are able to proliferate and maintain gut homeostasis. Further work, however, will be

395 needed to confirm our results which are largely predictive in nature.

396 Conclusions

397 In summary, we observed significant differences in the microbial community structures in 398 different regions of the intestinal tract of wild pigs. Of note, we found the Actinobacteria

399 microbiota of these wild pigs to be highly distinct from those of domesticated pigs subjected to

400 domestication, environmental and nutrient source changes, and artificial selection. While

401 Bifidobacterium and Allobaculum of these forms of bacteria are present within the intestines of

402 wild pigs, their densities are also much higher than that in domesticated animals. This indicates

403 that in the process of pig evolution, the intestinal microbes also evolved, and changes in the

404 intestinal microbial diversity are one of the main driving forces for the evolution of pigs.

405 Functional analyses of the intestinal microbiome in these wild pigs revealed that there were a

406 number of distinct metabolic pathways and spatial structures within this system. Overall, these

407 results offer unique insights that would help to advance the current understanding of how the

408 intestinal microbes interact with the host and affect the evolution of pigs.

409 


\section{Funding information}

412

This study was supported by the Foundation of He'nan Educational Committee of China

413 (14B230017), Industry University Research Cooperation Project of He'nan Science and

414 Technology Committee of China (182107000041), Science and Technology Opening

415 Cooperation Project of He'nan Science and Technology Committee of China (182106000039).

416 Conflict of interest

417 The authors declare no conflict of interest.

418

419

420

421

422

423

424

425

426

427

428

429

430

431

432

433

434

435

436

\section{References}

Brestoff JR, Artis D. 2013. Commensal bacteria at the interface of host metabolism and the immune system. Nat Immunology 14: 676-684.DOI: 10.1038/ni.2640.

Sharpton Thomas J .2018. Role of the gut microbiome in vertebrate evolution. mSystems 3(2): e00174-17.DOI: 10.1128/mSystems.00174-17

Mach N, Berri M, Estellé J, Levenez F, Lemonnier G, Denis C, Leplat JJ, Chevaleyre C, Billon Y, Doré J, Rogel-Gaillard C, Lepage P. 2015. Early-life establishment of the swine gut microbiome and impact on host phenotypes. Environmental Microbiology Reports 7: 554 569.DOI: 10.1111/1758-2229.12285.

Ramayo-Caldas Y, Mach N, Lepage P, Levenez F, Denis C, Lemonnier G, Leplat JJ, Billon Y, Berri M, Doré J, Rogel-Gaillard C, Estellé J .2016. Phylogenetic network analysis applied to pig gut microbiota identifies an ecosystem structure linked with growth traits. ISME Journal 10(12): 2973-2977. DOI: 10.1038/ismej.2016.77.

McCormack UM, Curião T, Buzoianu SG, Prieto ML, Ryan T, Varley P, Crispie F, Magowan E, Metzler-Zebeli BU, Berry D, O'Sullivan O, Cotter PD, Gardiner GE, Lawlor PG. 2017. Exploring a possible link between the intestinal microbiota and feed efficiency in pigs. Applied and Environmental Microbiology 83(15): e00380-17. DOI:10.1128/AEM.0038017.

Quan J, Cai G, Yang M, Zeng Z, Ding R, Wang X, Zhuang Z, Zhou S, Li S, Yang H, Li Z, Zheng E, Huang W, Yang J, Wu Z. 2019. Exploring the fecal microbial composition and metagenomic functional capacities associated with feed efficiency in commercial DLY pigs. Frontiers in Microbiology 10:52. DOI: 10.3389/fmicb.2019.00052.

Quan J, Cai G, Ye J, Yang M, Ding R, Wang X, Zheng E, Fu D, Li S, Zhou S, Liu D, Yang J, $\mathrm{Wu} Z$. 2018. A global comparison of the microbiome compositions of three gut locations in commercial pigs with extreme feed conversion ratios. Scientific Reports 8(1):4536. DOI: 10.1038/s41598-018-22692-0. genome influence on gut microbial composition and microbial prediction of complex traits in pigs. Genetics 206(3):1637-1644. DOI: 10.1534/genetics.117.200782.

Crespo-Piazuelo D, Estellé J, Revilla M, Criado-Mesas L, Ramayo-Caldas Y, Óvilo C, 
449

450

451

452

453

454

455

456

457

458

459

460

461

462

463

464

465

466

467

468

469

470

471

472

473

474

475

476

477

478

479

480

481

482

483

484

485

486

487

Fernández AI, Ballester M, Folch JM. 2018. Characterization of bacterial microbiota compositions along the intestinal tract in pigs and their interactions and functions. Scientific Reports 8(1): 12727. DOI:10.1038/s41598-018-30932-6.

Gao P, Liu Y, Le B, Qin B, Liu M, Zhao Y, Guo X, Cao G, Liu J, Li B, Duan Z. 2019. A comparison of dynamic distributions of intestinal microbiota between Large White and Chinese Shanxi Black pigs. Arch Microbiology 201(3):357-367. DOI: 10.1007/s00203019-01620-4.

Kelly J, Daly K, Moran AW, Ryan S, Bravo D, Shirazi-Beechey SP. 2017. Composition and diversity of mucosa-associated microbiota along the entire length of the pig gastrointestinal tract; dietary influences. Environmental Microbiology 19(4):1425-1438. DOI:10.1111/1462-2920.13619.

Xiao Y, Kong F, Xiang Y, Zhou W, Wang J, Yang H, Zhang G, Zhao J. 2018. Comparative biogeography of the gut microbiome between jinhua and landrace pigs. Scientific Reports 8(1): 5985. DOI: 10.1038/s41598-018-24289-z.

Larson G, Dobne K, Albarella U, Fang M, Matisoo-Smith E, Robins J, Lowden S, Finlayson H, Brand T, Willerslev E, Rowley-Conwy P, Andersson L, Cooper A. 2005. Worldwide phylogeography of wild boar reveals multiple centers of pig domestication. Science 307(5715): 1618-1621. DOI: 10.1126/science.1106927.

Yang GL, Shi CX, Fu DL, Li ZQ .2019. The mutations within MC1R, TYRP1, ASIP genes and their effects on phenot ypes of coat color in wild pigs (Sus scrofa ussuricus). Indian Journal of Animal Research 53(3): 288-293. DOI: 10.18805/ijar.B-804.

Brooks AW, Kohl KD, Brucker RM, van Opstal EJ, Bordenstein SR. 2016. Phylosymbiosis: relationships and functional effects of microbial communities across host evolutionary history. Plos Biology 14(11):e2000225. DOI: 10.1371/journal.pbio.2000225.

Gaulke CA, Arnold HK, Kembel SW, O’Dwyer JP, Sharpton TJ. 2017. Ecophylogenetics reveals the evolutionary associations between mammals and their gut microbiota. bioRxiv https://DOI.org/10.1101/ 182212.

Groussin M, Mazel F, Sanders JG, Smillie CS, Lavergne S, Thuiller W, Alm EJ. 2017. Unraveling the processes shaping mammalian gut microbiomes over evolutionary time. Nature Communications 8:14319. DOI: 10.1038/ncomms14319.

Ley RE, Hamady M, Lozupone C, Turnbaugh PJ, Ramey RR, Bircher JS, Schlegel ML, Tucker TA, Schrenzel MD, Knight R, Gordon JI. 2008. Evolution of mammals and their gut microbes. Science 320(5883):1647-1651. DOI: 10.1126/science.1155725.

Koskella B, Hall LJ, Metcalf CJE. 2017. The microbiome beyond the horizon of ecological and evolutionary theory. Nature Ecology \& Evolution 1(11):1606-1615. DOI:10.1038/s41559017-0340-2.

Moeller AH, Caro-Quintero A, Mjungu D, Georgiev AV, Lonsdorf EV, Muller MN, Pusey AE, Peeters M, Hahn BH, Ochman H. 2016. Cospeciation of gut microbiota with hominids. Science 3353(6297):380-382. DOI: 10.1126/science.aaf3951.

Peer] reviewing PDF | (2019:09:41671:2:0:NEW 31 Mar 2020) 
488

489

490

491

492

493

494

495

496

497

498

499

500

501

502

503

504

505

506

507

508

509

510

511

512

513

514

515

516

517

518

519

520

521

522

523

524

525

526

Muegge BD, Kuczynski J, Knights D, Clemente JC, González A, Fontana L, Henrissat B, Knight $\mathrm{R}$, Gordon JI. 2011. Diet drives convergence in gut microbiome functions across mammalian phylogeny and within humans. Science 332(6032):970-974. DOI:10.1126/science.1198719 .

Nayfach S, Bradley PH, Wyman SK, Laurent TJ, Williams A, Eisen JA, Pollard KS, Sharpton TJ. 2015. Automated and accurate estimation of gene family abundance from shotgun metagenomes. PLoS Computational Biology 11(11):e1004573.

DOI:10.1371/journal.pcbi.1004573.

Rosshart SP, Vassallo BG, Angeletti D, Hutchinson DS, Morgan AP, Takeda K, Hickman HD, McCulloch JA, Badger JH, Ajami NJ, Trinchieri G, Pardo-Manuel de Villena F, Yewdell JW, Rehermann B. 2017. Wild mouse gut microbiota promotes host fitness and improves disease resistance. Cell 171(5):1015-1028.e13. DOI:10.1016/j.cell.2017.09.016.

Kohl KD, Weiss RB, Cox J, Dale C, Dearing MD. 2014. Gut microbes of mammalian herbivores facilitate intake of plant toxins. Ecology Letters 17(10):1238-1246. DOI:10.1111/ele.12329.

Ushida K, Tsuchida S, Ogura Y, Toyoda A, Maruyama F. 2016. Domestication and cereal feeding developed domestic pig-type intestinal microbiota in animals of suidae. Animal Science Journal 87(6):835-841. DOI: 10.1111/asj.12492.

Caporaso JG, Kuczynski J, Stombaugh J, Bittinger K, Bushman FD, Costello EK, Fierer N, Peña AG, Goodrich JK, Gordon JI, Huttley GA, Kelley ST, Knights D, Koenig JE, Ley RE, Lozupone CA, McDonald D, Muegge BD, Pirrung M, Reeder J, Sevinsky JR, Turnbaugh PJ, Walters WA, Widmann J, Yatsunenko T, Zaneveld J, Knight R. 2010. QIIME allows analysis of high-throughput community sequencing data. Nat Methods 7(5):335-336. DOI:10.1038/nmeth.f.303.

Magoc T, Salzberg SL. 2011. FLASH: fast length adjustment of short reads to improve genome assemblies. Bioinformatics 27(21):2957-2963. DOI:10.1093/bioinformatics/btr507.

Edgar RC, Haas BJ, Clemente JC, Quince C, Knight R. 2011. UCHIME improves sensitivity and speed of chimera detection. Bioinformatics 27(16):2194-2200.

DOI:10.1093/bioinformatics/btr381.

DeSantis TZ, Hugenholtz P, Larsen N, Rojas M, Brodie EL, Keller K, Huber T, Dalevi D, Hu P, Andersen GL. 2006. Greengenes, a chimera-checked 16S rRNA gene database and workbench compatible with ARB. Applied and Environmental Microbiology 72(7): 50695072. DOI: 10.1128/AEM.03006-05.

Lozupone C, Knight R. 2005. UniFrac: a new phylogenetic method for comparing microbial communities. Applied and Environmental Microbiology 71(12):8228-8235.

DOI:10.1128/AEM.71.12.8228-8235.2005.

Lozupone CA, Hamady M, Kelley ST, Knight R. 2007. Quantitative and qualitative beta diversity measures lead to di erent insights into factors that structure microbial communities. Applied and Environmental Microbiology 73: 1576-1585. DOI: 10.1128/AEM.01996-06.

Peer] reviewing PDF | (2019:09:41671:2:0:NEW 31 Mar 2020) 
527

528

529

530

531

532

533

534

535

536

537

538

539

540

541

542

543

544

545

546

547

548

549

550

551

552

553

554

555

556

557

558

559

560

561

562

563

564

565

Ramette A .2007. Multivariate analyses in microbial ecology. Fems Microbiology Ecology 62(2): 142-160. DOI: 10.1111/j.1574-6941.2007.00375.x.

White JR, Nagarajan N, Pop M. 2009. Statistical methods for detecting differentially abundant features in clinical metagenomic samples. PLoS Computational Biology 5(4):e1000352. DOI: 10.1371/journal.pcbi.1000352.

Segata N, Izard J, Waldron L, Gevers D, Miropolsky L, Garrett WS, Huttenhower C. 2011. Metagenomic biomarker discovery and explanation. Genome Biology 12(6): R60. DOI:10.1186/gb-2011-12-6-r60.

Langille MG, Zaneveld J, Caporaso JG, McDonald D, Knights D, Reyes JA, Clemente JC, Burkepile DE, Vega Thurber RL, Knight R, Beiko RG, Huttenhower C. 2013. Predictive functional profiling of microbial communities using 16S rRNA marker gene sequences. Nature Biotechnology 31(9):814-821. DOI: 10.1038/nbt.2676.

Yang H, Huang X, Fang S, Xin W, Huang L, Chen C. 2016. Uncovering the composition of microbial community structure and metagenomics among three gut locations in pigs with distinct fatness. Scientific Reports 6:27427. DOI: 10.1038/srep27427.

Zhao W, Wang Y, Liu S, Huang J, Zhai Z, He C, Ding J, Wang J, Wang H, Fan W, Zhao J, Meng H. 2015. The dynamic distribution of porcine microbiota across different ages and gastrointestinal tract segments. PLoS One 10(20): e0117441.

DOI:10.1371/journal.pone.0117441.

He M, Fang S, Huang X, Zhao Y, Ke S, Yang H, Li Z, Gao J, Chen C, Huang L. 2016. Evaluating the contribution of gut microbiota to the variation of porcine fatness with the cecum and fecal samples. Frontiers in Microbiology 7:2108.

DOI:10.3389/fmicb.2016.02108.

Ivarsson E, Roos S, Liu HY, Lindberg JE. 2014. Fermentable non-starch polysaccharides increases the abundance of Bacteroides-Prevotella-Porphyromonas in ileal microbial community of growing pigs. Animal 8: 1777-1787. DOI:10.1017/s1751731114001827.

Kraler M, Ghanbari M, Domig KJ, Schedle K, Kneifel W. 2016. The intestinal microbiota of piglets fed with wheat bran variants as characterised by $16 \mathrm{~S}$ rRNA next-generation amplicon sequencing. Archives of Animal Nutrition 70(3): 173-189.

DOI:10.1080/1745039X.2016.1160534.

Liu H, Ivarsson E, Dicksved J, Lundh T, Lindberg JE. 2012. Inclusion of Chicory. Cichorium intybus L. in pigs' diets affects the intestinal microenvironment and the gut microbiota. Applied and Environmental Microbiology 78: 4102-4109. DOI:10.1128/AEM.07702-11. DOI:10.1128 / AEM.01996-06.

Slifierz MJ, Friendship RM, Weese JS. 2015. Longitudinal study of the early-life fecal and nasal microbiotas of the domestic pig. BMC Microbiol 15(1):184. DOI: 10.1186/s12866-0150512-7.

Mell J C, Redfield RJ. 2014. Natural competence and the evolution of DNA uptake specificity. Journal of Bacteriology 196, 1471-1483. DOI: 10.1128/JB.01293-13. 
566 Lammerts van Bueren A, Saraf A, Martens E C, Dijkhuizen L. 2015. Differential metabolism of

567 exopolysaccharides from probiotic Lactobacilli by the human gut symbiont Bacteroides

568 thetaiotaomicron. Applied and Environmental Microbiology 81(12): 3973-3983.DOI:

569

570 10.1128/AEM.00149-15.

Matsui T, Tanaka J, Namihira T, Shinzato N. 2012. Antibiotics production by an actinomycete

571

572

573

574

575

576

577

578

579

580

581

582

583

584

585

586

587

588

589

590

591

592

593

594

595

596

597

598

599

600

601

602

603

604 isolated from the termite gut. Journal of Basic Microbiology 52(6):731-735. DOI:10.1002/jobm.201100500.

Xiao Y, Li K, Xiang Y, Zhou W, Gui G, Yang H. 2017. The fecal microbiota composition of boar Duroc, Yorkshire, Landrace and Hampshire pigs. Asian Australas. Journal of Animal Science 30: 1456-1463. DOI: 10.5713/ajas.16.0746.

Furusawa Y, Obata Y, Fukuda S, Endo TA, Nakato G, Takahashi D, Nakanishi Y, Uetake C, Kato K, Kato T, Takahashi M, Fukuda NN, Murakami S, Miyauchi E, Hino S, Atarashi K, Onawa S, Fujimura Y, Lockett T, Clarke JM, Topping DL, Tomita M, Hori S, Ohara O, Morita T, Koseki H, Kikuchi J, Honda K, Hase K, Ohno H. 2013. Commensal microbederived butyrate induces the differentiation of colonic regulatory $\mathrm{T}$ cells. Nature 504(7480):446-450. DOI: 10.1038/nature12721.

Rigo-Adrover M, Saldaña-Ruíz S, van Limpt K, Knipping K, Garssen J, Knol J, Franch A, Castell M, Pérez-Cano FJ. 2017. A combination of scGOS/lcFOS with Bifidobacterium breve M-16V protects suckling rats from rotavirus gastroenteritis. European Journal Of Nutrition 56(4):1657-1670. DOI: 10.1007/s00394-016-1213-1.

Satoh T, Izumi H, Iwabuchi N, Odamaki T, Namba K, Abe F, Xiao JZ. 2016. Bifidobacterium breve prevents necrotising enterocolitis by suppressing inflammatory responses in a preterm rat model. Benef Microbes 7(1):75-82. DOI: 10.3920/BM2015.0035.

Wu SF, Chiu HY, Chen AC, Lin HY, Lin HC, Caplan M. 2013. Efficacy of different probiotic combinations on death and necrotizing enterocolitis in a premature rat model. Journal of Pediatric Gastroenterology and Nutrition 57(1):23-28.

DOI:10.1097/MPG.0b013e3182929210.

Izumi H, Minegishi M, Sato Y, Shimizu T, Sekine K, Takase M. 2015. Bifidobacterium breve alters immune function and ameliorates DSS-induced inflammation in weanling rats. Pediatric Research 78(4):407-416. DOI: 10.1038/pr.2015.115.

Sadeghzadeh J, Vakili A, Sameni HR, Shadnoush M, Bandegi AR, Zahedi Khorasani M. 2017. The effect of oral consumption of probiotics in prevention of heart injury in a rat myocardial infarction model: a histopathological, hemodynamic and biochemical evaluation. Iran Biomed J 21(3): 174-181. DOI:10.18869/acadpub.ibj.21.3.174.

Bordoni A, Amaretti A, Leonardi A, Boschetti E, Danesi F, Matteuzzi D, Roncaglia L, Raimondi S, Rossi M. 2013. Cholesterol-lowering probiotics: In vitro selection and in vivo testing of bifidobacteria. Applied Microbiology and Biotechnology 97(18): 8273-8281. DOI: 10.1007/s00253-013-5088-2.

Kim SJ, Park SH, Sin HS, Jang SH, Lee SW, Kim SY, Kwon B, Yu KY, Kim SY, Yang DK. 
605

606

607

608

609

610

611

612

613

614

615

616

617

618

619

620

621

622

623

624

625

626

627

628

629

630

631

632

633

634

635

636

637

638

639

640

641

2017. Hypocholesterolemic effects of probiotic mixture on diet-induced hypercholesterolemic Rats. Nutrients 9(3): E293. DOI: 10.3390/nu9030293.

Plaza-Díaz J, Plaza-Díaz J, Robles-Sánchez C, Abadía-Molina F, Sáez-Lara MJ, Vilchez-Padial LM, Gil Á, Gómez-Llorente C, Fontana L. 2017a. Gene expression profiling in the intestinal mucosa of obese rats administered probiotic bacteria. Scientific Data 4:170186. DOI: $10.1038 /$ sdata.2017.186.

Plaza-Díaz J, Robles-Sánchez C, Abadía-Molina F, Morón-Calvente V, Sáez-Lara MJ, RuizBravo A, Jiménez-Valera M, Gil Á, Gómez-Llorente C, Fontana L. 2017b. Adamdec1, Ednrb and Ptgs1/Cox1, inflammation genes upregulated in the intestinal mucosa of obese rats, are down regulated by three probiotic strains. Scientific Reports 7(1):1939. DOI: 10.1038/s41598-017-02203-3.

Plaza-Diaz J, Gomez-Llorente C, Abadia-Molina F, Saez-Lara MJ, Campaña-Martin L, MuñozQuezada S, Romero F, Gil A, Fontana L. 2014. Effects of Lactobacillus paracasei CNCM I4034, Bifidobacterium breve CNCM I-4035 and Lactobacillus rhamnosus CNCM I-4036 on hepatic steatosis in Zucker rats. PLoS One 9(50): e98401. DOI:10.1371/journal.pone.0098401.

Zhu G, Ma F, Wang G, Wang Y, Zhao J, Zhang H, Chen W. 2018. Bifidobacteria attenuate the development of metabolic disorders, with inter- and intra-species differences. Food \& Function 9(6):3509-3522. DOI: 10.1039/c8fo00100f.

Baldwin J, Collins B, Wolf PG, Martinez K, Shen W, Chuang CC, Zhong W, Cooney P, Cockrell C, Chang E, Gaskins HR, McIntosh MK. 2016. Table grape consumption reduces adiposity and markers of hepatic lipogenesis and alters gut microbiota in butter fat-fed mice. Journal of Nutritional Biochemistry 27:123-135. DOI:10.1016/j.jnutbio.2015.08.027.

Tsuchida S, Maruyama F, Ogura Y, Toyoda A, Hayashi T, Okuma M and Ushida K. 2017. Genomic characteristics of Bifidobacterium thermacidophilum pig isolates and wild boar isolates reveal the unique presence of a putative mobile genetic element with tetW for pig farm isolates. Frontiers in Microbiology 8:1540. DOI:10.3389/fmicb.2017.01540.

Niu Q, Li P, Hao S, Zhang Y, Kim SW, Li H, Ma X, Gao S, He L, Wu W, Huang X, Hua J, Zhou B, Huang R. 2015. Dynamic distribution of the gut microbiota and the relationship with apparent crude fiber digestibility and growth stages in pigs. Scientific Reports 5: 9938. DOI: $10.1038 /$ srep09938.

Yang H, Huang X, Fang S, He M, Zhao Y, Wu Z, Yang M, Zhang Z, Chen C, Huang L. 2017. Unraveling the fecal microbiota and metagenomic functional capacity associated with feed efficency in pigs. Front Microbiol 8, 1555. DOI:10.3389/fmicb.2017.01555.

Pryde SE, Duncan SH, Hold GL, Stewart CS, Flint HJ. 2002. The microbiology of butyrate formation in the human colon. Fems Microbiology Letters 217(2): 133-139. DOI:10.1111/j.1574-6968.2002.tb11467.x. 


\section{Figure 1}

Species accumulation (A) and rank-abundance (B) curves analysis of the different gut intestinal tract samples at $97 \%$ sequences identity.

Fig. 1 Species accumulation (A) and rank-abundance (B) curves analysis of the different gut intestinal tract samples at $97 \%$ sequences identity. Duodenum (DU), jejunum (JE), ileum (IL), cecum (CE), and colon (CO). If the curves reach or nearly reach a plateau, it suggests that most of the species present in all samples have been observed.

(A)

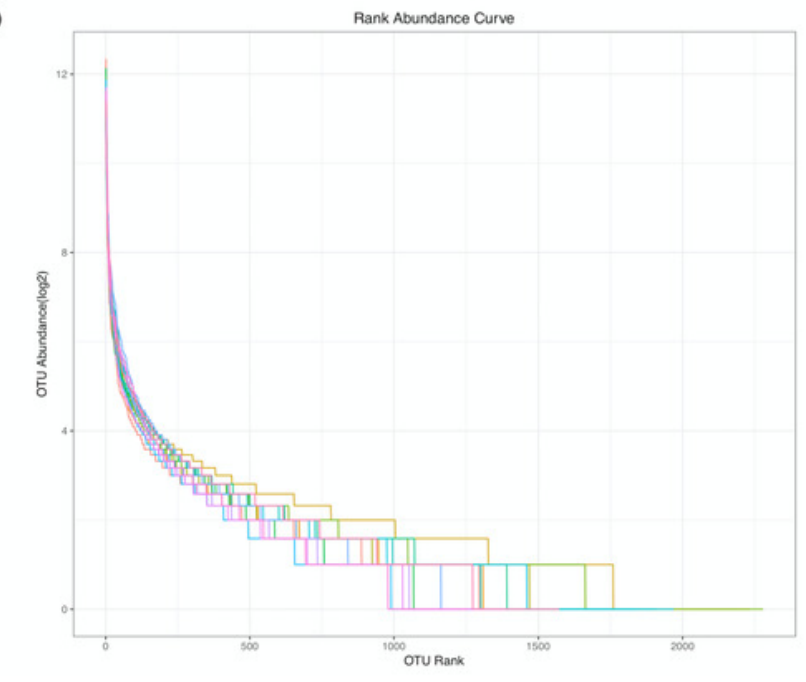

(B)

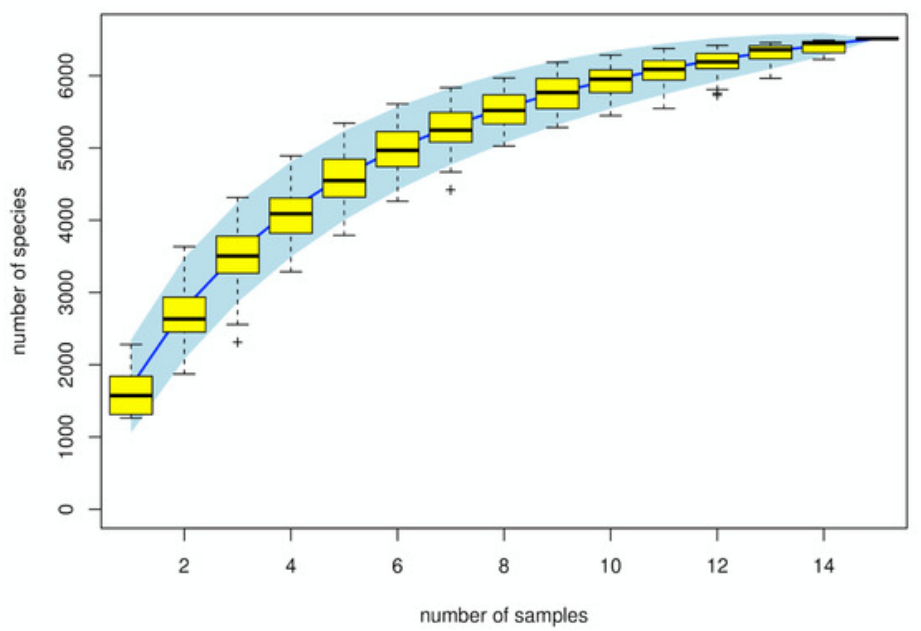


Figure 2

The alpha-diversity comparisons for the duodenum (DU), jejunum (JE), ileum (IL), cecum (CE), and colon (CO).

Fig. 2 The alpha-diversity comparisons for the duodenum (DU), jejunum (JE), ileum (IL), cecum (CE), and colon (CO). (A) The ACE index at the sampling location (mean \pm SD). (B) The Chaol index at the sampling location (mean \pm SD). (C) The Shannon's diversity index at the sampling location (mean \pm SD). (D) The Simpson index at the sampling location (mean \pm $\mathrm{SD})$.
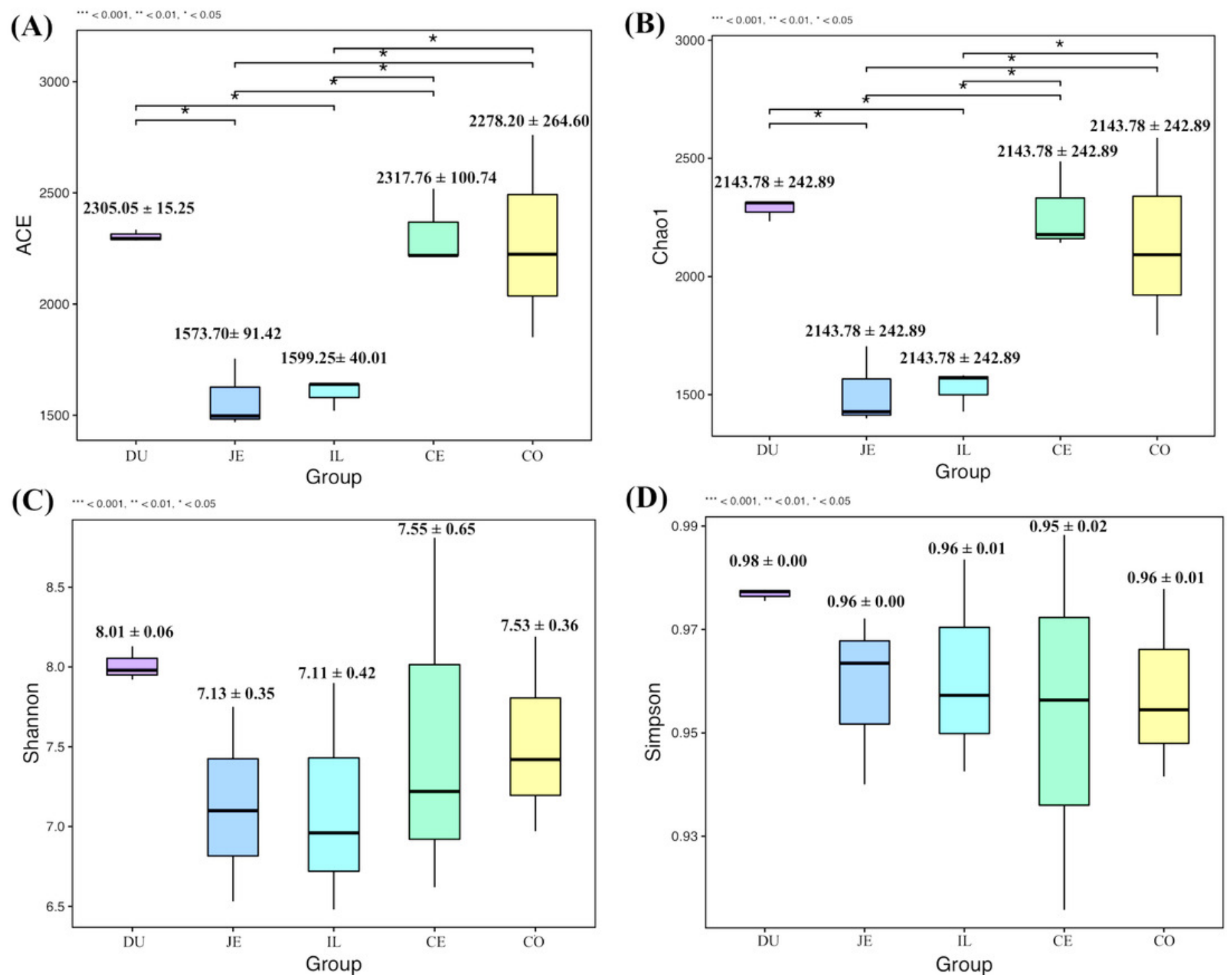
Figure 3

The beta-diversity comparisons for the duodenum (DU), jejunum (JE), ileum (IL), cecum (CE), and colon (CO).

Fig. 3 The beta-diversity comparisons for the duodenum (DU), jejunum (JE), ileum (IL), cecum (CE), and colon (CO). (A) Unweighted UniFrac NMDS of the microbiota. Each symbol and color denote each gut location microbiota. (B) Bray Curtis dendrogram analyses were performed on the 16S rRNA V3-V4 region.

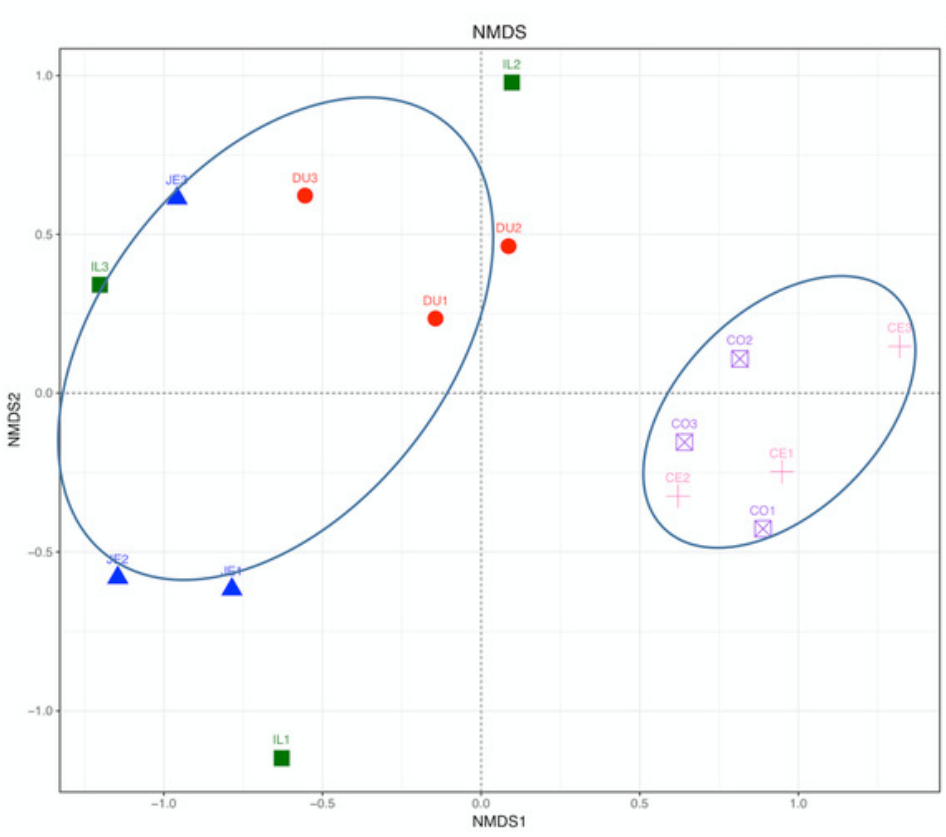

(A)

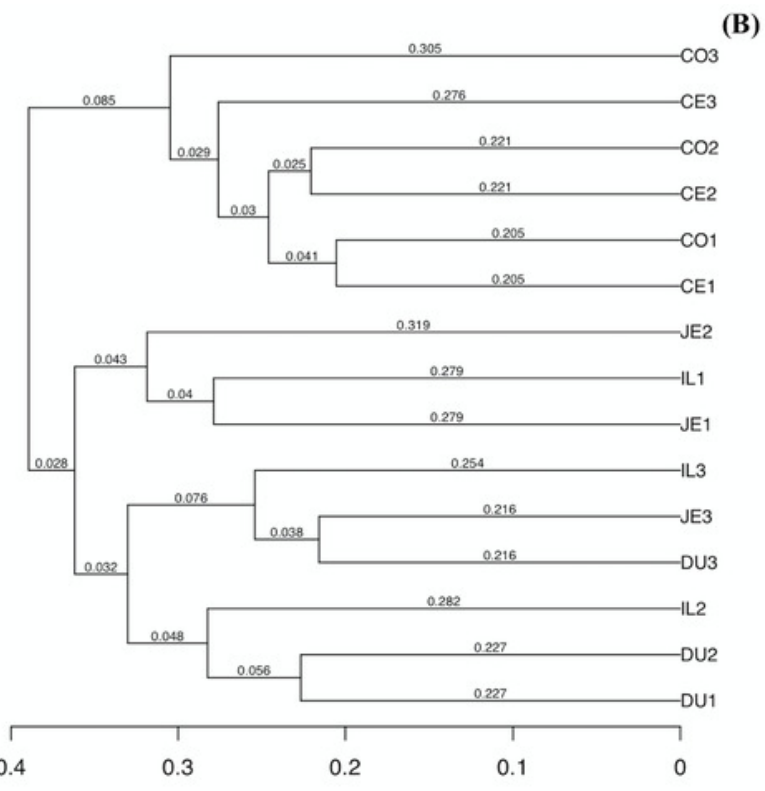




\section{Figure 4}

Community composition of the gut microbiota in different intestinal segments of wild pigs at the phylum (A) and genus (B) levels, respectively.

Fig. 4 Community composition of the gut microbiota in different intestinal segments of wild pigs at the phylum (A) and genus (B) levels, respectively. Duodenum (DU), jejunum (JE), ileum(IL), cecum(CE), and colon (CO).
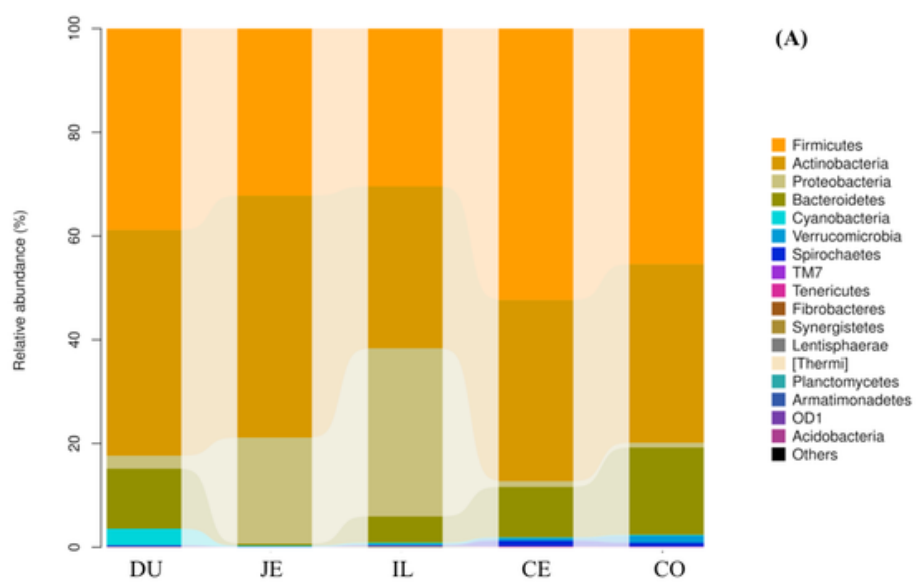
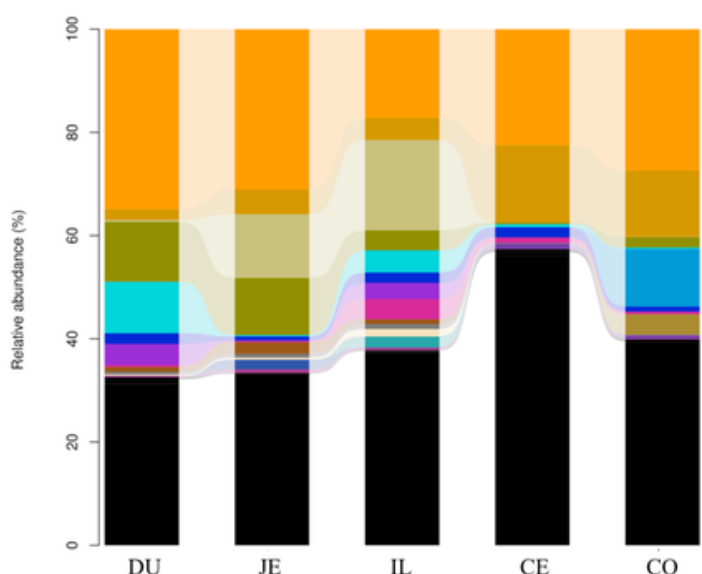

(B)

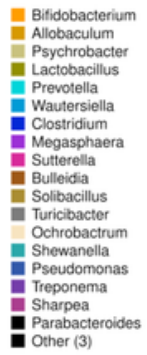

IL 


\section{Figure 5}

Bacterial taxa differentially represented in ileum (IL), cecum (CE), and colon (CO) gut locations in wild pigs identified by LEFSe using an LDA score threshold of $>2.0$.

Fig. 5 Bacterial taxa differentially represented in ileum (IL), cecum (CE), and colon (CO) gut locations in wild pigs identified by LEFSe using an LDA score threshold of $>2.0$.

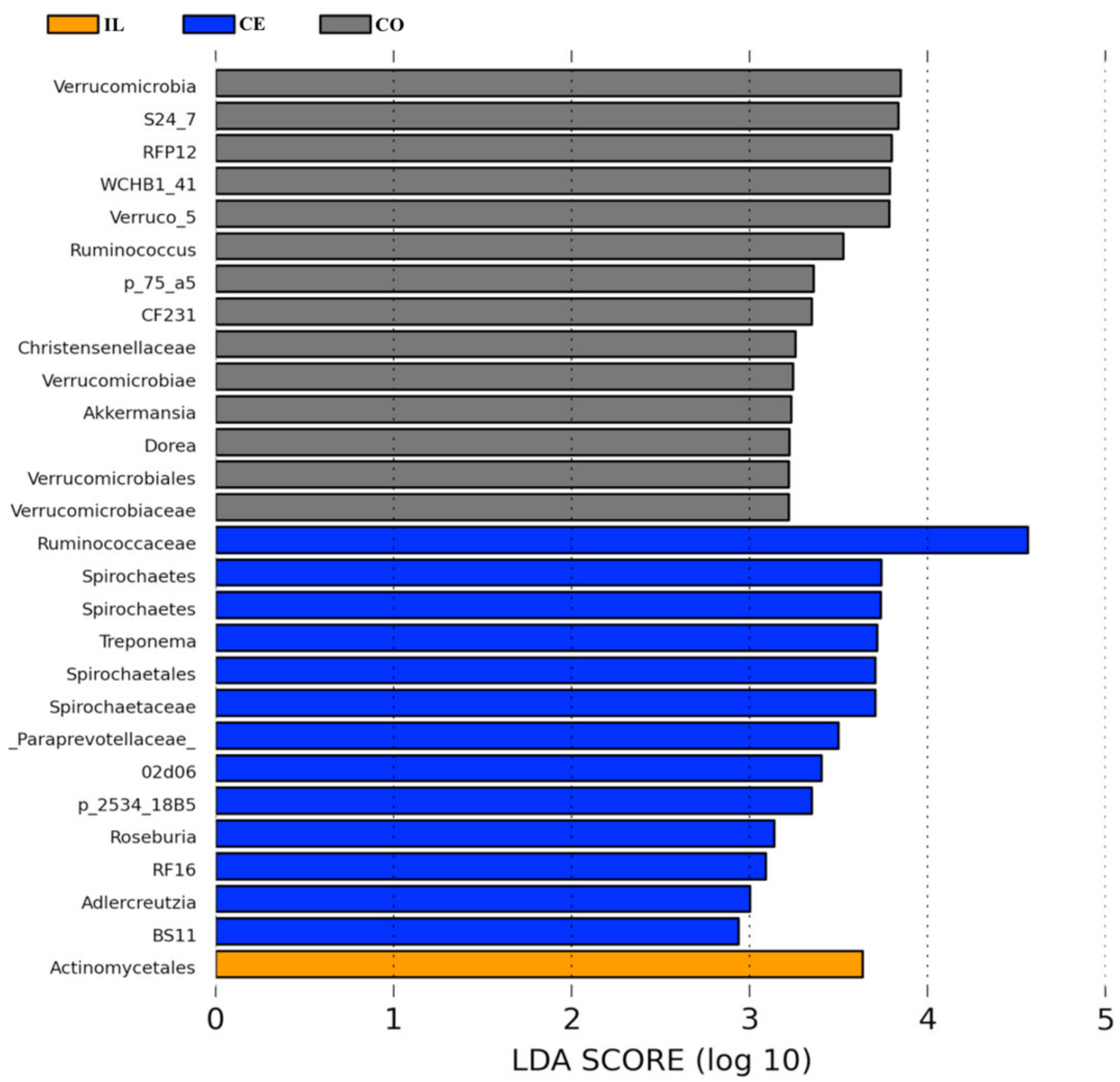




\section{Figure 6}

Predicted functional of the gut microbiota in the duodenum (DU), jejunum (JE), ileum (IL), cecum (CE), and colon (CO).

Fig. 6 Predicted functional of the gut microbiota in the duodenum (DU), jejunum (JE), ileum (IL), cecum (CE), and colon (CO). The vertical columns represent groups, and the horizontal rows depict metabolic pathways. The color coding is based on row z-scores.

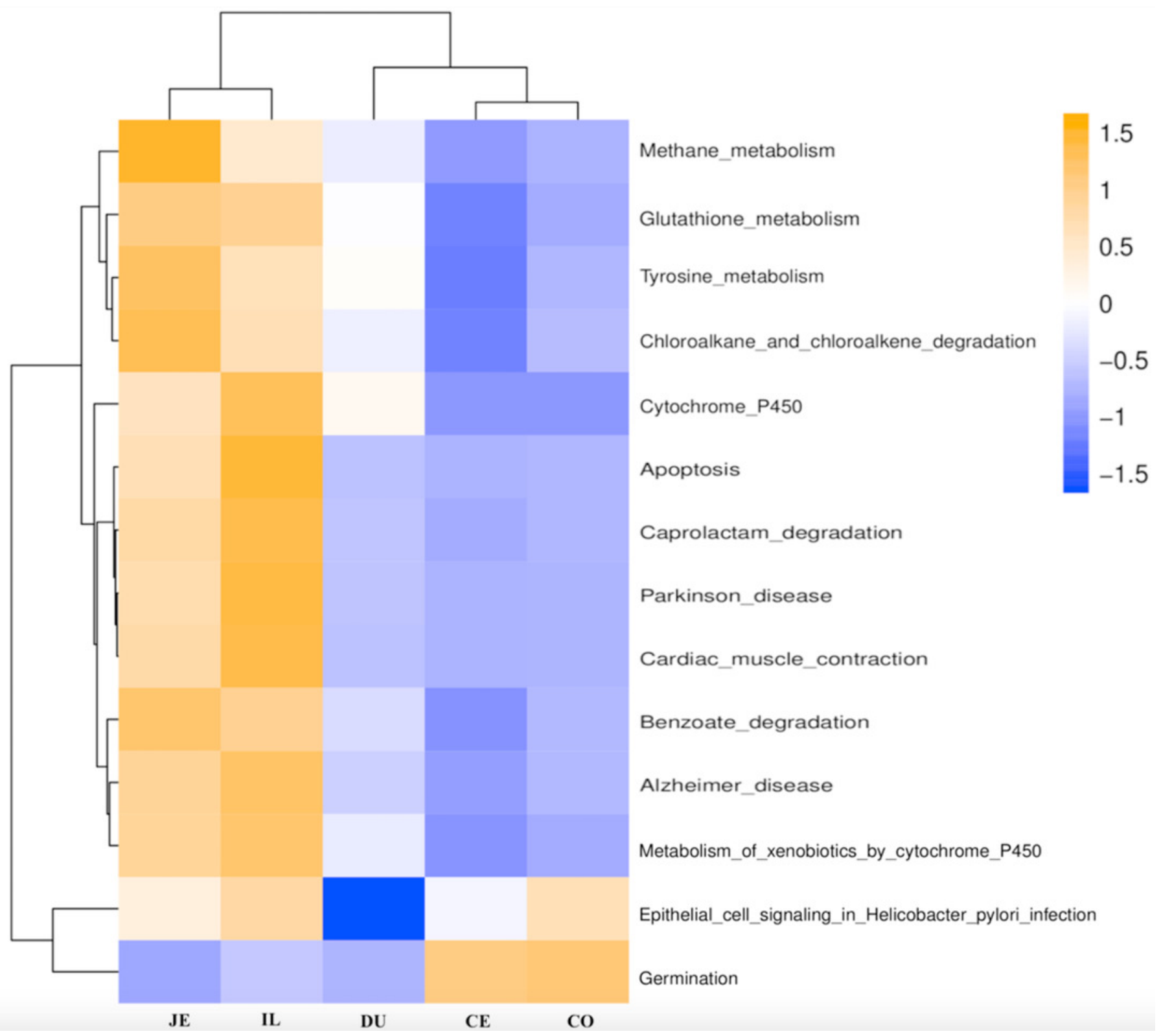




\section{Figure 7}

FPredicted functional differentially of the bacterial genus represented in the jejunum (JE), ileum (IL), and cecum (CE) gut locations in wild pigs identified by LEFSe using an LDA score threshold of $>2.0$.

Fig. 7 Predicted functional differentially of the bacterial genus represented in the jejunum (JE), ileum (IL), and cecum (CE) gut locations in wild pigs identified by LEFSe using an LDA score threshold of $>2.0$.

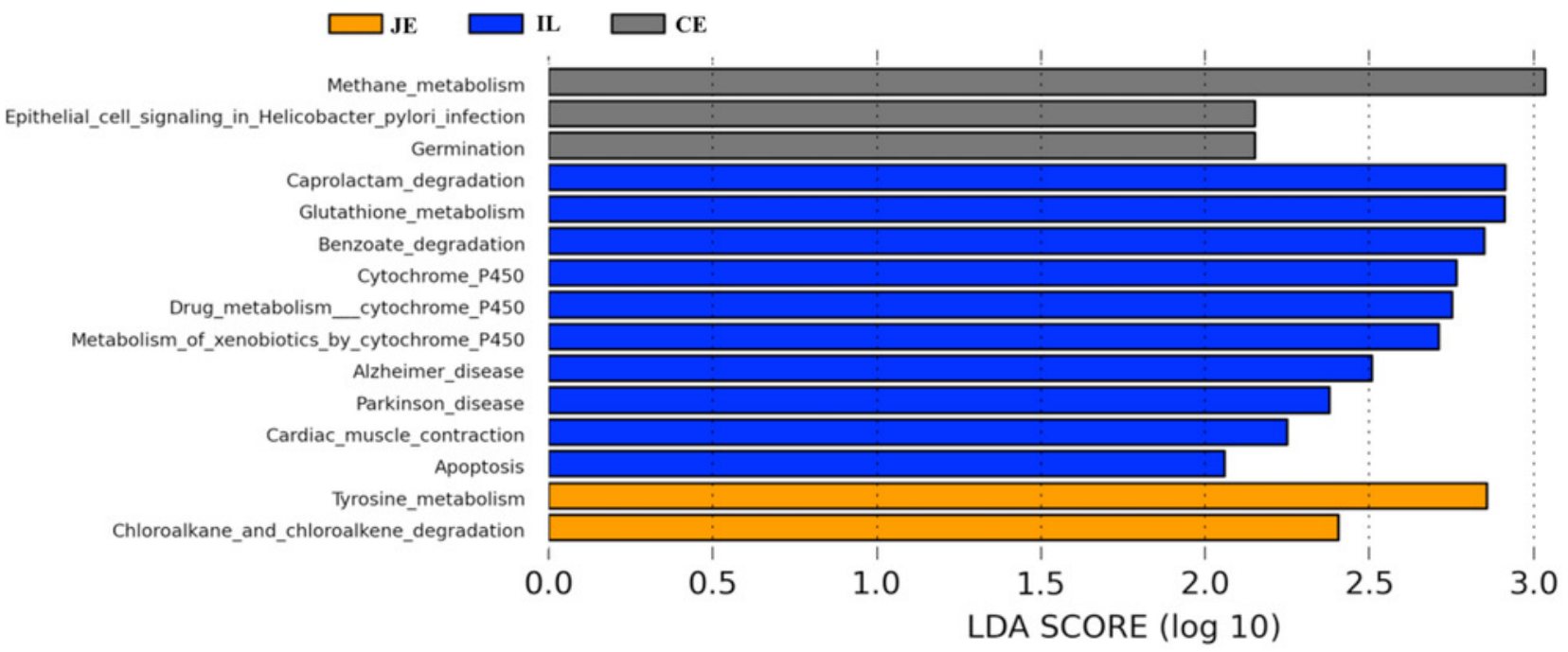




\section{Figure 8}

Heatmap clustered by the KEGG pathway showing different enrichments in the duodenum (DU), jejunum (JE), ileum (IL), cecum (CE), and colon (CO) of wild pigs.

Fig. 8 Heatmap clustered by the KEGG pathway showing different enrichments in the duodenum (DU), jejunum (JE), ileum (IL), cecum (CE), and colon (CO) of wild pigs. The vertical columns represent groups, and the horizontal rows depict metabolic pathways. The color coding is based on row z-scores.

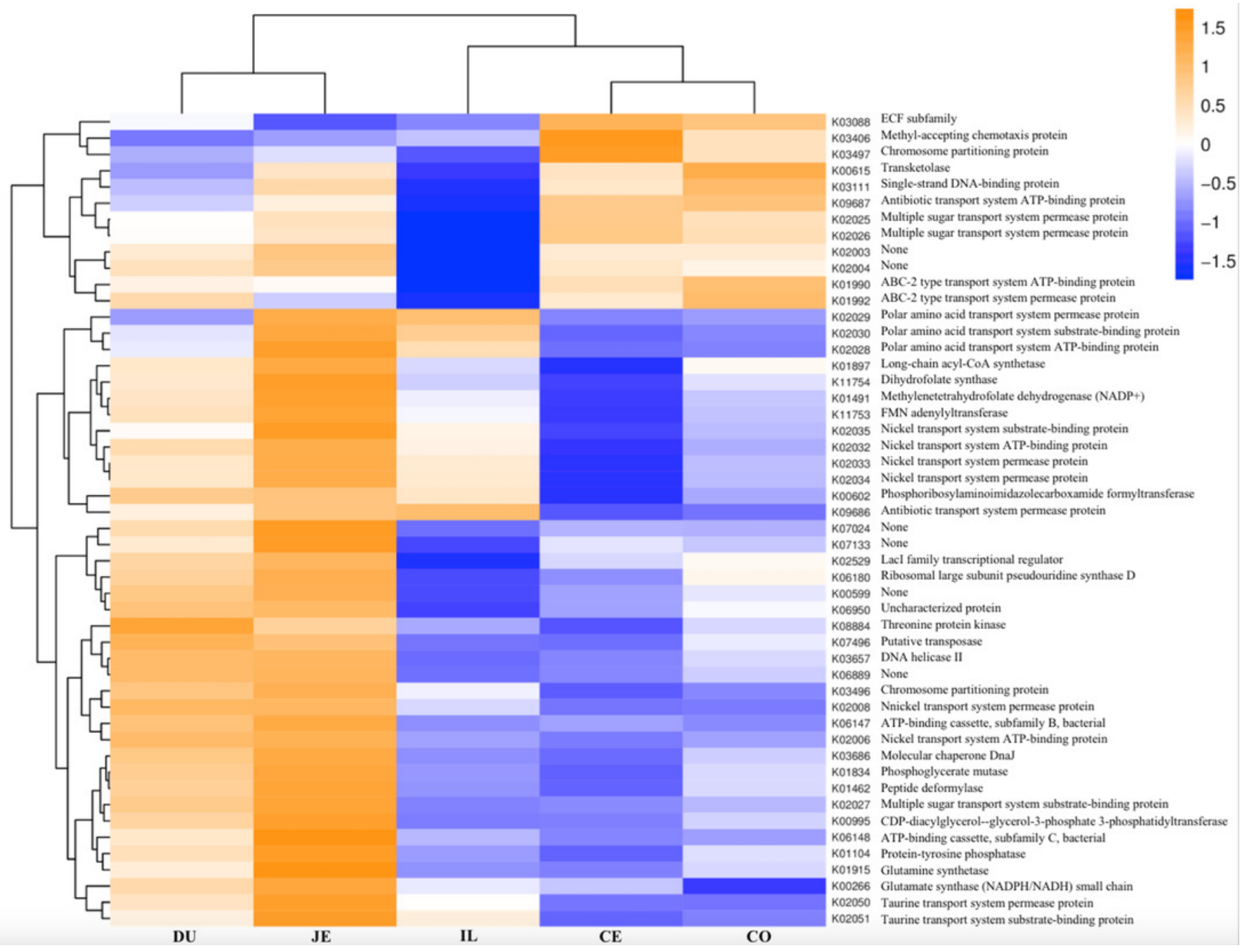

